\title{
EXCITON BINDING ENERGY IN EXTREMELY SHALLOW QUANTUM WELLS
}

\author{
J. KossuT \\ Institute of Physics, Polish Academy of Sciences \\ Al. Lotników 32/46, 02-668 Warszawa, Poland \\ AND J.K. FURDYNA
}

Department of Physics, University of Notre Dame, Notre Dame, IN 46556, USA

The usual approach to the problem of excitons in semiconductor quantum wells is to assume that both the electron or the hole are primarily localized in the potential well regions defined by the band offsets, i.e., that the quantum wells are deep. We re-examine the problem of the exciton in the presence of a very shallow square well potential due to the (small) conduction and valence band offsets in a semiconducting heterostructure. We show that the combined effects of the shallow well and the Coulomb interaction between the electron and the hole are equivalent to an effective potential acting on the center-of-mass of a three-dimensional exciton. We calculate the shape of such a potential and show it to be satisfactorily approximated by the potential of a parabolic well.

PACS numbers: 73.20.Dx, 71.36.+c, 73.61.Ga

The existing variational theories of excitons in quasi two-dimensional semiconductor structures most often rely on the assumption that the exciton wave function is primarily localized in the quantum well (usually taken as a square well) region defined by the conduction and the valence band offsets. Consequently, the trial wave function of an exciton in a quasi two-dimensional structure is assumed to have a general form proportional to a product of $\chi\left(z_{\mathrm{e}}\right)$ and $\chi\left(z_{\mathrm{h}}\right)$, where $\chi\left(z_{\mathrm{e}}\right)$ represents the wave function of the (ground) state of an electron localized in the square potential well, and $\chi\left(z_{\mathrm{h}}\right)$ - that of the valence band hole. This product is then multiplied by an excitonic ground state envelope factor $\mathrm{e}^{-r / \lambda}$, where $r$ is the relative coordinate of the electron and the hole, and $\lambda$ is a two-dimensional exciton radius treated as a variational parameter [1].

If the wells for either the holes or for the electrons are shallow, the validity of the above assumption - and thus the applicability of the above simple trial wave function - break down. In many realistic situations involving II-VI semiconductors this is actually the case. In particular, in studies of spin superlattices [2] — by the very design of these structures - the "deep well" approach just 
described is not applicable. Various ways out of this difficulty were proposed in the literature [3-7]. The majority of them are formulated for the case where one of the particles forming the exciton (e.g., the electron) is still strongly localized, while the other (e.g., the hole) is not. Quite recently, a more general approach was constructed, where neither the hole nor the electron are strongly bound by the confining potentials due to the band discontinuities [8]. Unfortunately, the latter approach relies on a lengthy variational self-consistent calculation that is physically not very transparent. Therefore, in the present paper, an attempt is made to find an analytical solution of the problem keeping the physics of the situation explicitly in focus.

In our analysis we will specifically consider the type I configuration. For simplicity, we shall consider only heavy holes. The masses of the particles in question are denoted by $m_{\mathrm{e}}$ and $m_{\mathrm{h}}$. The Hamiltonian of the problem reads

$$
\mathcal{H}_{\mathrm{ex}}=E_{\mathrm{g}}+\frac{p_{\mathrm{e}}{ }^{2}}{2 m_{\mathrm{e}}}+\frac{p_{\mathrm{h}}{ }^{2}}{2 m_{\mathrm{h}}}+V_{\mathrm{e}}\left(z_{\mathrm{e}}\right)+V_{\mathrm{h}}\left(z_{\mathrm{h}}\right)-\frac{e^{2}}{\varepsilon\left|r_{\mathrm{e}}-r_{\mathrm{h}}\right|} .
$$

We denoted the value of the energy gap in the barrier material by $E_{\mathrm{g}}$. The potentials due to band edge discontinuities are

$$
V_{\mathrm{e}}\left(z_{\mathrm{e}}\right)=\left\{\begin{array}{cl}
-V_{\mathrm{e}}^{0} & \text { if } w / 2<z_{\mathrm{e}} \leq w / 2 \\
0 & \text { otherwise, }
\end{array}\right.
$$

( $w$ being the width of quantum well) and

$$
V_{\mathrm{h}}\left(z_{\mathrm{h}}\right)=\left\{\begin{array}{cl}
V_{\mathrm{h}}^{0} & \text { if } w / 2<z_{\mathrm{e}} \leq w / 2 \\
0 & \text { otherwise. }
\end{array}\right.
$$

We assume that $V_{\mathrm{e}}^{0}$ and $V_{\mathrm{h}}^{0}$ are positive quantities.

Introducing, as usually, the center-of-mass and the relative position variables and substituting them in Eq. (1) we obtain

$$
\mathcal{H}_{\mathrm{ex}}=E_{\mathrm{g}}+\frac{P_{\perp}^{2}}{2 M}+\frac{P_{Z}^{2}}{2 M}+\left(\frac{\pi^{2}}{2 \mu}-\frac{e^{2}}{\varepsilon r}\right)+V_{\mathrm{e}}\left(Z+\frac{m_{\mathrm{h}}}{M} z\right)+V_{\mathrm{h}}\left(Z-\frac{m_{\mathrm{e}}}{M} z\right),
$$

where $M$ and $\mu$ is the center-of-mass and reduced mass of the exciton, respectively. In the above expression $\pi$ denotes the momentum operator corresponding to the relative variable $\boldsymbol{r}$, while $\boldsymbol{P}_{\perp}$ and $P_{Z}$ are the components (two-dimensional and one-dimensional, respectively) of the momentum corresponding to the center-of-mass variable. The second term describes the kinetic energy of the center-of-mass motion in the layer plane, while the third - that of the motion normal plane of the well. The term in parentheses corresponds to the three-dimensional relative motion of an exciton. The two remaining terms mix the center-of-mass and the relative position variables. If these two terms are "weak" (we shall discuss later this assumption in more quantitative terms), one can expect the three-dimensional character of the exciton wave function to be only slightly perturbed. It is, therefore, reasonable to assume the approximate ground state solutions of Eq. (4) in the form

$$
\psi_{\mathrm{ex}}(\boldsymbol{R}, \boldsymbol{r})=\frac{1}{\sqrt{S}} \mathrm{e}^{\mathrm{i} \boldsymbol{K}_{\perp} \cdot \boldsymbol{\Lambda}} \frac{1}{\sqrt{\pi} a_{\mathrm{B}}^{3}} \mathrm{e}^{-r / a_{\mathrm{B}}} F(Z),
$$

where $S$ is the area of the sample, $a_{\mathrm{B}}$ is the radius of a three-dimensional exciton, $\boldsymbol{A}$ is the two-dimensional coordinate vector for the free-like in-plane motion of the 
exciton center-of-mass and $\boldsymbol{K}_{\perp}$ is the corresponding momentum vector. The function $F(Z)$ (yet to be determined) is normalized to unity. Calculating $\left\langle\psi_{\mathrm{ex}}\left|\mathcal{H}_{\mathrm{ex}}\right| \psi_{\mathrm{ex}}\right\rangle$, one obtains for the energy of an exciton

$$
\begin{aligned}
& \left\langle\psi_{\mathrm{ex}}\left|\mathcal{H}_{\mathrm{ex}}\right| \psi_{\mathrm{ex}}\right\rangle=E_{\mathrm{g}}+\varepsilon_{3 \mathrm{D}}+\frac{\hbar^{2} K_{\perp}^{2}}{2 M}+\langle F(Z)| \frac{P_{Z}^{2}}{2 M} \\
& +\frac{1}{\pi a_{\mathrm{B}}^{3}} \int \mathrm{e}^{-2 r / a_{\mathrm{B}}}\left[V_{\mathrm{e}}\left(Z+m_{\mathrm{h}} / M z\right)+V_{\mathrm{h}}\left(Z-m_{\mathrm{e}} / M z\right)\right] \mathrm{d}^{3} r|F(Z)\rangle .
\end{aligned}
$$

Here $\varepsilon_{3 \mathrm{D}}$ is the energy of the three-dimensional exciton $\left(\varepsilon_{3 \mathrm{D}}=-\mu e^{4} / 2 \varepsilon^{2} \hbar^{2}\right)$ and $a_{\mathrm{B}}=\hbar^{2} \varepsilon / \mu e^{2}$ is its radius. After performing the integration over the three-dimensional relative variable $r$ the operators in the last term in Eq. (6) can be treated as an effective Hamiltonian which determines the function $F(Z)$,

$$
\mathcal{H}^{\mathrm{eff}}(Z) F(Z)=\frac{P_{Z}^{2}}{2 M}+V_{\mathrm{e}}^{\mathrm{eff}}(Z)+V_{\mathrm{h}}^{\mathrm{eff}}(Z)=\varepsilon^{\prime} F(Z),
$$

where $\varepsilon^{\prime}$ gives the correction to the exciton energy due to the weak perturbation by the quantum well potentials. For the effective potential $V_{c}^{\text {eff }}$ originating in the square quantum well in the conduction band explicit integration gives

$$
\begin{aligned}
& V_{\mathrm{e}}^{\mathrm{eff}}(Z)=-\frac{1}{2} V_{\mathrm{e}}^{0}\left\{\left[-1-\left(\frac{w}{2}-Z\right) \frac{1}{a_{\mathrm{B}}} \frac{M}{m_{\mathrm{h}}}\right] \mathrm{e}^{-\frac{2}{a_{\mathrm{B}}}\left(\frac{w}{2}-Z\right) \frac{M}{m_{\mathrm{h}}}}\right. \\
& \left.+\left[1-\left(\frac{w}{2}+Z\right) \frac{1}{a_{\mathrm{B}}} \frac{M}{m_{\mathrm{h}}}\right] \mathrm{e}^{\frac{2}{a_{\mathrm{B}}}\left(\frac{w}{2}+Z\right) \frac{M}{m_{\mathrm{h}}}}\right\} \quad \text { for } Z<-\frac{w}{2}, \\
& V_{\mathrm{e}}^{\mathrm{eff}}(Z)=-\frac{1}{2} V_{\mathrm{e}}^{0}\left\{\left[-1-\left(\frac{w}{2}+Z\right) \frac{1}{a_{\mathrm{B}}} \frac{M}{m_{\mathrm{h}}}\right] \mathrm{e}^{-\frac{2}{a_{\mathrm{B}}}\left(\frac{w}{2}+Z\right) \frac{M}{m_{\mathrm{h}}}}\right. \\
& \left.+\left[-1-\left(\frac{w}{2}-Z\right) \frac{1}{a_{\mathrm{B}}} \frac{M}{m_{\mathrm{h}}}\right] \mathrm{e}^{-\frac{2}{a_{\mathrm{B}}}\left(\frac{w}{2}-Z\right) \frac{M}{m_{\mathrm{h}}}}+2\right\} \quad \text { for }-\frac{w}{2} \leq Z \leq \frac{w}{2}, \\
& V_{\mathrm{e}}^{\mathrm{eff}}(Z)=-\frac{1}{2} V_{\mathrm{e}}^{0}\left\{\left[-1-\left(\frac{w}{2}+Z\right) \frac{1}{a_{\mathrm{B}}} \frac{M}{m_{\mathrm{h}}}\right] \mathrm{e}^{-\frac{2}{a_{\mathrm{B}}}\left(\frac{w}{2}+Z\right) \frac{M}{m_{\mathrm{h}}}}\right. \\
& \left.+\left[1-\left(\frac{w}{2}-Z\right) \frac{1}{a_{\mathrm{B}}} \frac{M}{m_{\mathrm{h}}}\right] \mathrm{e}^{\frac{2}{a_{\mathrm{B}}}\left(\frac{w}{2}-Z\right) \frac{M}{m_{\mathrm{h}}}}\right\} \quad \text { for } Z>\frac{w}{2} .
\end{aligned}
$$

Similarly, for the valence band component of the effective potential we have

$$
\begin{aligned}
& V_{\mathrm{h}}^{\mathrm{eff}}(Z)=\frac{1}{2} V_{\mathrm{h}}^{0}\left\{\left[1+\left(\frac{w}{2}-Z\right) \frac{1}{a_{\mathrm{B}}} \frac{M}{m_{\mathrm{e}}}\right] \mathrm{e}^{-\frac{2}{a_{\mathrm{B}}}\left(\frac{w}{2}-Z\right) \frac{M}{m_{\mathrm{e}}}}\right. \\
& \left.+\left[-1+\left(\frac{w}{2}+Z\right) \frac{1}{a_{\mathrm{B}}} \frac{M}{m_{\mathrm{e}}}\right] \mathrm{e}^{\frac{2}{a_{\mathrm{B}}}\left(\frac{w}{2}+Z\right) \frac{M}{m_{\mathrm{e}}}}\right\} \quad \text { for } Z<-\frac{w}{2}, \\
& V_{\mathrm{h}}^{\mathrm{eff}}(Z)=\frac{1}{2} V_{\mathrm{h}}^{0}\left\{\left[1+\left(\frac{w}{2}-Z\right) \frac{1}{a_{\mathrm{B}}} \frac{M}{m_{\mathrm{e}}}\right] \mathrm{e}^{-\frac{2}{a_{\mathrm{B}}}\left(\frac{w}{2}-Z\right) \frac{M}{m_{\mathrm{e}}}}\right. \\
& \left.+\left[1+\left(\frac{w}{2}+Z\right) \frac{1}{a_{\mathrm{B}}} \frac{M}{m_{\mathrm{e}}}\right] \mathrm{e}^{-\frac{2}{a_{\mathrm{B}}}\left(\frac{w}{2}+Z\right) \frac{M}{m_{\mathrm{e}}}}-2\right\} \quad \text { for }-\frac{w}{2} \leq Z \leq \frac{w}{2} \text {, } \\
& V_{\mathrm{h}}^{\mathrm{eff}}(Z)=\frac{1}{2} V_{\mathrm{h}}^{0}\left\{\left[-1+\left(\frac{w}{2}-Z\right) \frac{1}{a_{\mathrm{B}}} \frac{M}{m_{\mathrm{e}}}\right] \mathrm{e}^{\frac{2}{a_{\mathrm{B}}}\left(\frac{w}{2}+Z\right) \frac{M}{m_{\mathrm{e}}}}\right. \\
& \left.+\left[1+\left(\frac{w}{2}+Z\right) \frac{1}{a_{\mathrm{B}}} \frac{M}{m_{\mathrm{e}}}\right] \mathrm{e}^{-\frac{2}{a_{\mathrm{B}}}\left(\frac{w}{2}+Z\right) \frac{M}{m_{\mathrm{e}}}}\right\} \quad \text { for } Z>\frac{w}{2} \text {. }
\end{aligned}
$$


It is interesting to note that the effective potential can be approximated quite accurately by a parabolic potential whose solutions can be easily found (strictly speaking, the approximation is valid if $w / a_{\mathrm{B}}$ is small; however, direct inspection shows that even for $w \approx a_{\mathrm{B}}$ the approximation is still quite accurate and physically realistic):

$$
V_{\mathrm{e}}^{\mathrm{eff}}(Z) \approx V_{\mathrm{e}}^{0}\left\{-1+\mathrm{e}^{-\frac{w}{a_{\mathrm{B}}} \frac{M}{m_{\mathrm{h}}}}\left[1+\frac{1}{2} \frac{w}{a_{\mathrm{B}}} \frac{M}{m_{\mathrm{h}}}+\frac{w}{a_{\mathrm{B}}^{3}}\left(\frac{M}{m_{\mathrm{h}}}\right)^{3} Z^{2}\right]\right\}
$$

and

$$
V_{\mathrm{h}}^{\mathrm{eff}}(Z) \approx V_{\mathrm{h}}^{0}\left\{-1+\mathrm{e}^{-\frac{w}{a_{\mathrm{B}}} \frac{M}{m_{\mathrm{e}}}}\left[1+\frac{1}{2} \frac{w}{a_{\mathrm{B}}} \frac{M}{m_{\mathrm{e}}}+\frac{w}{a_{\mathrm{B}}^{3}}\left(\frac{M}{m_{\mathrm{e}}}\right)^{3} Z^{2}\right]\right\} .
$$

The correction (close to the bottom of the bands) to the lowest energy of the nearly three-dimensional exciton weakly perturbed by the potential of the quantum well can be compactly expressed by

$$
\varepsilon^{\prime}=\varepsilon_{0}^{\prime}+\frac{1}{2} \hbar \omega,
$$

where

$$
\begin{aligned}
\varepsilon_{0}^{\prime}= & -\left(\left|V_{\mathrm{e}}^{0}\right|+\left|V_{\mathrm{h}}^{0}\right|\right)+\left|V_{\mathrm{e}}^{0}\right|\left(1+\frac{1}{2} \frac{w}{a_{\mathrm{B}}} \frac{M}{m_{\mathrm{h}}}\right) \mathrm{e}^{-\frac{w}{a_{\mathrm{B}}} \frac{M}{m_{\mathrm{h}}}} \\
& +\left|V_{\mathrm{h}}^{0}\right|\left(1+\frac{1}{2} \frac{w}{a_{\mathrm{B}}} \frac{M}{m_{\mathrm{e}}}\right) \mathrm{e}^{-\frac{w}{a_{\mathrm{B}}} \frac{M}{m_{\mathrm{e}}}}
\end{aligned}
$$

and

$$
\omega=\sqrt{2\left(\left|V_{\mathrm{e}}^{0}\right| \mathrm{e}^{-\frac{w}{a_{B}} \frac{M}{m_{\mathrm{h}}}} \frac{1}{m_{\mathrm{h}}^{3}}+\left|V_{\mathrm{h}}^{0}\right| \mathrm{e}^{-\frac{w}{a_{\mathrm{B}}} \frac{M}{m_{\mathrm{e}}}} \frac{1}{m_{\mathrm{e}}^{3}}\right) \frac{w M^{2}}{a_{B}^{3}}} .
$$

Substituting typical values of the material and structure parameters: $m_{\mathrm{e}}=0.1 m_{0}, m_{\mathrm{h}}=0.6 m_{0}, \varepsilon=10$ and $V_{\mathrm{e}}^{0}=V_{\mathrm{h}}^{0}=10 \mathrm{meV}$ we obtain for a $50 \AA$ quantum well $\varepsilon_{0}^{\prime}=-14.2 \mathrm{meV}$ and $\hbar \omega=2.3 \mathrm{meV}$. The assumed depths of the quantum well in the valence and the conduction band are smaller than the effective rydbergs for these bands (respectively, $81.6 \mathrm{meV}$ and $13.6 \mathrm{meV}$ ), which justifies the entire approach.

\section{Acknowledgments}

One of us would like to acknowledge the hospitality of the Department of Physics of the University of Notre Dame, where part of this study was completed. The study was supported in part by the U.S.-Poland Maria Curie-Skłodowska Joint Fund II under grant PAN/NSF-92-113 and partly by NSF grant DMR 9208400. Helpful discussions with Drs. Hong Luo, M. Dobrowolska and Guilin Yang are gratefully acknowledged. 


\section{References}

[1] See, e.g., G. Bastard, Wave Mechanics Applied to Semiconductor Heterostructures, Halstead Press, New York 1988, and references therein.

[2] N. Dai, H. Luo, F.C. Zhang, N. Samarth, M. Dobrowolska, J.K. Furdyna, Phys. Rev. Lett. 67, 3824 (1992); B.T. Jonker, X. Liu, W.C. Chou, A. Petrou, J. Warnock, J.J. Krebs, G.A. Prinz, J. Appl. Phys. 69, 6097 (1991).

[3] W.J. Walecki, A.V. Nurmikko, N. Samarth, H. Luo, J.K. Furdyna, N. Otsuka, Appl. Phys. Lett. 57, 466 (1990).

[4] C. Delalande, Superlattices Microstruct. 12, 387 (1992).

[5] E.I. Ivchenko, A.V. Kavokin, V.P. Kochereshko, G.R. Posina, I.N. Uraltsev, D.R. Yakovlev, R.N. Bicknell-Tassius, A. Waag, G. Landwehr, Phys. Rev. B 46, 7713 (1992).

[6] E. Delaporte, J.M. Berroir, G. Bastard, C. Delalande, J.M. Hong, L.L. Chang, Phys. Rev. B 42, 5891 (1990).

[7] Ji-Wei Wu, A.V. Nurmikko, Phys. Rev. B 38, 1504 (1988).

[8] J. Warnock, B.T. Jonker, A. Petrou, W.C. Chou, X. Liu, Phys. Rev. B 48, 17321 (1993). 